\title{
Automated and estimation methods to count nodule number in common bean
}

\author{
Polianna Alves Silva Dias ${ }^{1}$, Patrícia Guimarães Santos Melo ${ }^{1}$, Helton Santos Pereira ${ }^{2}$, Enderson \\ Petrônio de Brito Ferreira ${ }^{2 *}$
}

${ }^{1}$ Universidade Federal de Goiás, Rodovia GO-462, km 0, Campus Samambaia, Goiânia, GO, 74001-970, Brazil
${ }^{2}$ Embrapa Arroz e Feijão, Rodovia GO-462, km 12, Santo Antônio de Goiás, GO, 75375-000, Brazil

*Corresponding author: enderson.ferreira@embrapa.br

\begin{abstract}
The nodulation on legume roots is an important trait to evaluate the symbiotic efficiency, but very laborious and time spending, what can limit the effectiveness of legume breeding programs. Based on the need to simplify the assessment of nodule number, this work aimed to evaluate the accuracy and time spent to assess nodule number in common bean using different methods of estimation and counting, compared to manual count (MC), which was considered the standard method. The estimation methods consisted of two simple techniques with two sampling range: graph paper with sampling range from 10 to 20 nodules (GPR1) and graph paper with sampling range from 21 to 40 nodules (GPR2), Petri dish with sampling range from10 to 20 nodules (PDR1), Petri dish with sampling range from 21 to 40 nodules (PDR2). The counting was also performed using automated methods: the seeds counters Seedburo and Sanick. The time spent in each of the seven methods was recorded. Among the alternative methods, the automated counting methods stood out. The Seedburo 801 and Sanick ESC2011 counters showed high accuracy and reduced more than 50\% of the time spent compared to MC. Among the estimation methods, GPR2 showed good accuracy with 10\% reduction in time spent when compared to manual counting, being a good alternative when there are no automated counters.
\end{abstract}

Keywords: Phaseolus vulgaris; biological nitrogen fixation; nodulation; legume; counting methods.

\section{Introduction}

Biological nitrogen fixation (BNF) is a key process for the conversion of $\mathrm{N}$ gas $\left(\mathrm{N}_{2}\right)$ into ammonia $\left(\mathrm{NH}_{3}\right)$ performed by bacteria belonging to rhizobia group. The reduction of $\mathrm{N}_{2}$ to $\mathrm{NH}_{3}$ is carried out by $\mathrm{N}$-fixing bacteria or diazotrophic microorganisms containing the enzymatic complex, in which nitrogenase takes part (Novais et al., 2007). Among N-fixing bacteria of the rhizobia group, a variety of Rhizobium and Ensifer species is able to colonize and establish a symbiotic partnership with common bean (Dall'Agnol et al., 2013; Mhamdi et al, 2015).

The potential of the BNF to provide $\mathrm{N}$ for the plants has been recognized for many decades to several species, especially legumes. There are several traits that can be evaluated to infer the fixing ability of a given plant genotype or bacterial strain. Nodulation parameters, such as nodule number, nodule weight and, nodule activity, and $\mathrm{N}$ accumulation characters, like shoot $\mathrm{N}$ content and isotopic discrimination $\left({ }^{15} \mathrm{~N}\right)$ stand out among them. These traits are typically complementary since BNF process is complex. Among these traits, the number of nodules is widely used as evaluation and selection criteria of efficient plant genotypes and bacterial strains for BNF (Ferreira et al., 2000; Albuquerque et al., 2012; Barros et al., 2013; Fonseca et al., 2013).

The manual nodule counting is a simple and low-cost method widely used in Brazil. However, it is a very laborious technique, requiring great time consumption and intense concentration of the evaluator in order to avoid errors. Some attempts to simplify the evaluation of legumes nodulation have already been done. Lira Junior \& Smith (2000) used scanner methods of counting to assess nodule number in common bean, lentil and, pea. Based on their work, Lira Junior et al. (2003, 2005) and Costa et al. (2007) evaluated aspects of nodule development in common bean and other legumes using digital images. Also, Barbedo (2012) also used digital images to assess nodule number in legumes after the nodules have been removed from the roots. More recently, some efforts were made to use the minirhizotron imaging technique, a non-destructive procedure that originally allows the study of roots within the soil, to verify nodule development and senescence in soybean (Gray et al., 2013) and peanut (Rowland et al., 2015).

Using an estimation method, Cardoso et al. (2009) also tried to make easier the assessment of nodule number and weight. They found out that there is a relationship between the nodulation of the crown region and the whole root system in common bean, soybean and, peanut under Brazilian conditions. Despite their positive results, it is still necessary to count the nodules of the crown region.

Even though the limitation of time inherent to conventional nodulation determination might have been reduced, the use of scanners and digital images is a different approach, because these types of equipment might not be accessible to every breeding program working with BNF. Our goal was to reduce time in nodule evaluation with low cost and/or using equipment (as the seed counters) that are usual in a breeding program.

Although all the attempts, most of them using digital images, there are no efforts to obtain nodule number from simple estimates or using seeds counters, which are 
equipment routinely used in plant breeding programs for trials assembly. Neither there are studies that take into account the time spent on such evaluations, which is a limiting factor since in plant breeding and/or strains selection programs the number of lines/strains evaluated is usually large.

Based on the foregoing, this work aimed to evaluate the accuracy and time consumption to assess nodule number in common bean using different counting and estimation methods.

\section{Results}

\section{Determination of the nodule number by manual counting}

The goal of this work was not to determine the differences of nodule number among the common bean genotypes, but the accuracy and time spending of different methods applied on the counting and estimation of the nodule number. The nodule number per genotype, obtained by manual counting, varied from 31 to 263 (Table 1), which means, approximately 10 to 88 nodules per plant, reflecting the genotypes variability in response to inoculation.

\section{Accuracy of the alternative methods as compared to manual counting}

The comparison of manual with the alternative counting methods performed by $X^{2}$ test indicated that only the seed counters Seedburo ${ }^{\circledR}$ and Sanick ${ }^{\circledR}$ showed nodule number statistically identical to the obtained by the manual counting (MC) ( $>0.5$; Table 2). The other estimating methods, graph paper with sampling from 10 to 20 (GPR1) and from 21 to 40 (GPR2) nodules and, Petri dish with sampling from 10 to 20 (PDR1) and from 21 to 40 (PDR2) nodules, did not match with the number obtained manually $(\mathrm{p} \leq 0.01$; Table 2$)$.

According to mean error estimates, obtained from the errors of each genotype, both seed counters were highly accurate, with errors lower than $11 \%$ (Table 2). Sanick counter deserves evidence, with a mean error of only $4.4 \%$ (Table 2), with an amplitude between the genotypes from 0.0 to $14.3 \%$ (Table 1), confirming the high accuracy of this equipment. Among the estimation methods, GPR1 showed mean error very high $(63.7 \%)$ and also great variation among the genotypes (from 0.0 to $468.9 \%$, Table 1), indicating low accuracy. The other methods showed mean errors of $19.0 \%$ (GPR2), 26.8\% (PDR2) and 29.4\% (PDR1) (Table 2). Among these, GPR2 is noteworthy, for it showed mean error of $19.0 \%$ (Table 2) and lower variation amplitude between the errors of each genotype (1.2 to $65.1 \%$, Table 1 ).

Regarding Pearson and Spearman correlations, the results were similar. The nodule number obtained by the four estimating methods and both automated counters were strongly correlated with the nodule number obtained by manual counting $\left(\mathrm{p} \leq 0.01 ; \mathrm{r}\right.$ and $\left.\mathrm{r}_{\mathrm{s}} \geq 0.80^{*}\right)$. The exception was the GPR1, which showed intermediate Pearson correlation $(r=0.49$, Table 2). The GPR2 method and the seed counters are in evidence since these methods showed correlations above 0.94 (Table 2).

The analyses of coincidence and Hamblin \& Zimmermann (1986) coincidence index (CI) also showed identical results among the methods (Table 2). The coincidence was maximal for the GPR2 method and Sanick counter. The coincidence was high $(80 \%$, Table 2) for the PDR2 method and Seedburo counter. For the other methods, they would be coincidentally selected three out of five lines selected by MC $(60 \%$ coincidence, Table 2).
The coincidence index corresponds to the efficiency of the presumed lines selection by different methods, disregarding chance. Like for the coincidence, the CI was maximum for GPR2 method and Sanick counter (Table 2). Afterward, PDR2 method and Seedburo counter with the proportion of $73 \%$ of superior lines (Table 2).

\section{Time-saving associated to each evaluation method}

Another relevant information is the time spent on nodule counting, which, as expected, varied according to the nodule number of the different genotypes. Total time spent on the counting of the 20 genotypes using each method also varied, from 22.4 (Sanick counter) to 60.4 minutes (MC) (Table 3). The $X^{2}$ test for time showed that all six alternative methods were faster than manual counting ( $p \leq 0.01$; Table 2$)$, which is very interesting because represents a significant time-saving in evaluation. In relation to manual counting, the total time spent with the estimation methods (GPR1, GPR2, PDR1 and, PDR2) varied from $88 \%$ to $90 \%$. Using the seed counters the same evaluation would be held in half the time $(49 \%$ for Seedburo counter) and in only $37 \%$ of the time considering Sanick counter (Table 3 ).

\section{Discussion}

The determination of the nodulation ability of different genotypes of leguminous plants is crucial for the success of plant breeding programs aiming at the selection of efficient lines for BNF. For this process, the used methods must be fast and accurate. Evaluating commercial cultivars during the winter season, Pelegrin et al. (2009) and Fonseca et al. (2013) obtained an average of 12.6 and 15 nodules per plant when inoculated with $R$. tropici, respectively. This means that the average obtained in this study is in accordance with the literature. Considering that each sample was a line in evaluation for BNF, they would be selected the same five lines for GPR2 method and Sanick counter, when compared to the selection based on the results of manual counting. For the PDR2 method and Seedburo counter, four of the five supposed lines would match with the selection based on manual counting. These estimates of coincidence (of $80 \%$ or higher) are considered excellent, since the difference between the fifth and sixth genotypes with more nodules is only two nodules (167 and 169, Table 1), being, therefore, difficult to detect. Based on the seven criteria used to evaluate the accuracy of the estimation/counting methods (Table 2), Sanick and Seedburo seed counters stood out since they returned good results for all criteria. Seed counters are designed to count different sizes of seeds, so, if the nodules are properly dried, the seed counters showed to be a great option in nodule counting.

Besides the Sanick and Seedburo, GPR2 has also good accuracy, showing good estimates in six out of seven evaluated criteria. Assuming this is an estimation method, not a counting one, it is expected a higher error when compared to counting methods. Yet, GPR1 and GPR2 are alternative methods based on very accessible material, easy to make, and can be especially important when there are no available seed counters.

Lira Junior \& Smith (2000), that used scanner methods, obtained high correlation coefficients (from 0.85 to 0.98 ) between the automatic procedures and hand-counted nodule number, being an option for nodule counting if you have the appropriate material. Barbedo (2012), that also used digital images, obtained a good correlation between automatic and manual counting $(0.91)$. 
Table 1. Nodule number obtained per sample by manual counting and by the six estimation/counting methods.

\begin{tabular}{|c|c|c|c|c|c|c|c|}
\hline Genotype/Methods & $\mathrm{MC}^{1}$ & $\mathrm{PDR}^{2}{ }^{2}$ & $\mathrm{PDR}^{3}$ & $\mathrm{GPR}^{4}$ & $\mathrm{GPR}^{5}$ & Seedburo $^{6}$ & Sanick $^{7}$ \\
\hline GEN P5-4-3-1 & 31 & 70 & 45 & 40 & 39 & 16 & 32 \\
\hline CNFP 15188 & 40 & 45 & 39 & 43 & 51 & 36 & 38 \\
\hline CNFP 15193 & 42 & 40 & 71 & 75 & 49 & 42 & 48 \\
\hline CNFC 15082 & 53 & 67 & 59 & 53 & 58 & 51 & 51 \\
\hline CNFP 15175 & 68 & 100 & 79 & 71 & 82 & 67 & 65 \\
\hline CNFP 15207 & 77 & 95 & 86 & 63 & 90 & 71 & 68 \\
\hline CNFP 15194 & 78 & 59 & 57 & 72 & 65 & 63 & 76 \\
\hline CNFC 15010 & 83 & 124 & 143 & 137 & 137 & 78 & 80 \\
\hline CNFP 15208 & 83 & 88 & 102 & 79 & 107 & 60 & 81 \\
\hline IPR Uirapuru & 83 & 95 & 68 & 65 & 84 & 83 & 82 \\
\hline CNFP 15198 & 86 & 65 & 78 & 83 & 95 & 76 & 82 \\
\hline CNFP 15178 & 92 & 110 & 105 & 109 & 125 & 86 & 92 \\
\hline CNFP 15177 & 116 & 179 & 86 & 556 & 114 & 114 & 109 \\
\hline Pérola & 144 & 197 & 183 & 189 & 130 & 137 & 154 \\
\hline BRS CNFC 10425 & 167 & 135 & 296 & 950 & 159 & 159 & 164 \\
\hline BRS CNFC 10762 & 169 & 160 & 199 & 106 & 215 & 153 & 168 \\
\hline CNFC 15025 & 172 & 206 & 192 & 233 & 200 & 145 & 185 \\
\hline BRS Estilo & 181 & 95 & 179 & 139 & 197 & 170 & 172 \\
\hline BRS CNFC 10429 & 241 & 182 & 366 & 258 & 308 & 255 & 244 \\
\hline BRS Campeiro & 263 & 269 & 273 & 349 & 283 & 236 & 258 \\
\hline Total & 2,269 & 2,381 & 2,706 & 3,670 & 2,588 & 2,098 & 2,249 \\
\hline $\operatorname{Min}$ Error $^{8}(\%)$ & - & 2.3 & 1.1 & 0.0 & 1.2 & 0.0 & 0.0 \\
\hline $\operatorname{Max}_{\operatorname{Error}^{9}}(\%)$ & - & 125.8 & 77.2 & 468.9 & 65.1 & 48.4 & 14.3 \\
\hline
\end{tabular}
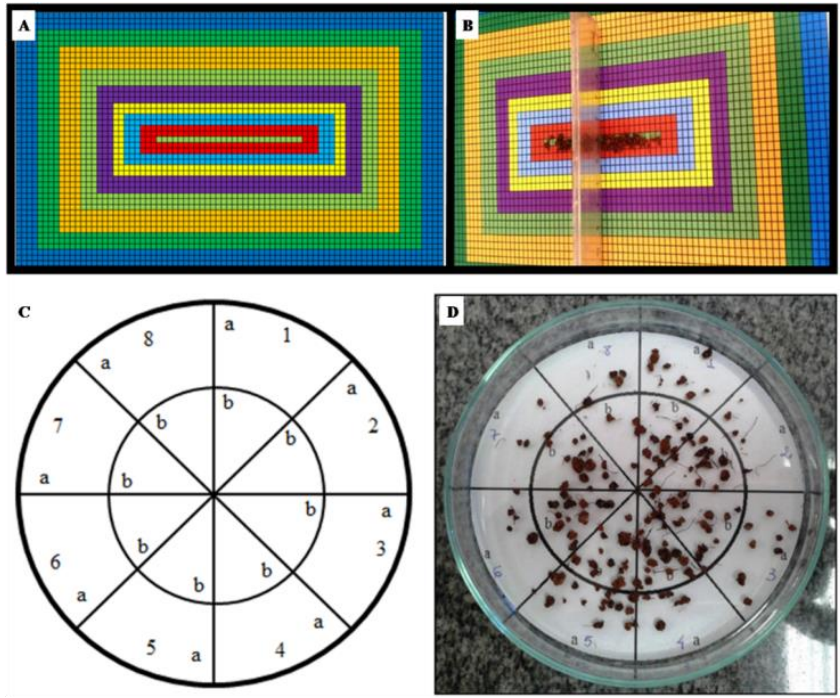

Fig 1. Graph paper sectored in colors for easy viewing of the nodules and the central region of the paper (A) and nodules distribution in the longitudinal axis of the paper and the use of the ruler for reducing nodules until the desired sampling range (B). Petri dish divided into quadrants (C) and sections with random distribution of nodules after gentle agitation (D).

Table 2. Results of the seven parameters used to verify the accuracy of the six nodule number estimation/counting methods, compared to the manual counting.

\begin{tabular}{|c|c|c|c|c|c|c|}
\hline Parameter & $\mathrm{PDR}^{1}{ }^{1}$ & $\mathrm{PDR}^{2}$ & $\mathrm{GPR}^{3}$ & $\mathrm{GPR}^{4}$ & Seedburo $^{5}$ & Sanick $^{6}$ \\
\hline$X^{2}$ nodule number & $230.77^{* *}$ & $279.88^{* *}$ & $5514.54^{* * *}$ & $109.54^{* * *}$ & $30.09^{\text {ns }}$ & $5.39^{\mathrm{ns}}$ \\
\hline Mean error $(\%)$ & 29.4 & 26.8 & 63.7 & 19.0 & 10.1 & 4.4 \\
\hline Pearson correlation & $0.84^{* *}$ & $0.91^{* * *}$ & $0.49^{* *}$ & $0.97^{* *}$ & $0.99^{* *}$ & $1.00^{* *}$ \\
\hline Spearman correlation & $0.80^{* *}$ & $0.87^{* * *}$ & $0.85^{* *}$ & $0.94^{* *}$ & $0.97^{* * *}$ & $1.00^{* *}$ \\
\hline Coincidence (\%) & 60 & 80 & 60 & 100 & 80 & 100 \\
\hline Coincidence index $(\%)$ & 47 & 73 & 47 & 100 & 73 & 100 \\
\hline$X^{2}$ time & $1,066^{* *}$ & $1,195^{* *}$ & $1,211^{* *}$ & $1,167^{* *}$ & $1,041^{* *}$ & $1,545^{* *}$ \\
\hline
\end{tabular}

significant $(\mathrm{p}<0.01)$ and ${ }^{\mathrm{ns}}$ non-significant $(\mathrm{p}>0.05)$ by $X^{2}$ test and by Student-t test (correlations).

${ }^{1}$ Petri dish estimation method with sampling range from 10 to 20 nodules; ${ }^{2}$ Petri dish estimation method with sampling range from 21 to 40 nodules; ${ }^{3} \mathrm{Graph}$ paper estimation method with sampling range from 10 to 20 nodules; ${ }^{4}$ Graph paper estimation method with sampling range from 21 to 40 nodules; ${ }^{5}$ Automated counting method by the seed counter Seedburo 801 cont-a-pak®; ${ }^{6}$ Automated counting method by the seed counter Sanick ESC $2011 \circledR$. 
Table 3. Time (seconds) spent on manual counting and on the six nodule number counting/estimation methods of three plants per genotype.

\begin{tabular}{|c|c|c|c|c|c|c|c|}
\hline Genotype/Methods & $\mathrm{MC}^{1}$ & $\mathrm{PDR}^{2}$ & $\mathrm{PDR}^{3}{ }^{3}$ & $\mathrm{GPR}^{4}{ }^{4}$ & $\mathrm{GPR}^{5}{ }^{5}$ & Seedburo $^{6}$ & Sanick $^{7}$ \\
\hline GEN P5-4-3-1 & 52 & 145 & 181 & 183 & 170 & 53 & 20 \\
\hline CNFP 15188 & 51 & 171 & 163 & 169 & 167 & 72 & 20 \\
\hline CNFP 15193 & 97 & 169 & 153 & 155 & 160 & 61 & 24 \\
\hline CNFC 15082 & 88 & 173 & 159 & 179 & 142 & 53 & 46 \\
\hline CNFP 15175 & 124 & 170 & 158 & 170 & 169 & 66 & 34 \\
\hline CNFP 15207 & 93 & 163 & 173 & 129 & 179 & 72 & 60 \\
\hline CNFP 15194 & 133 & 154 & 182 & 161 & 187 & 80 & 52 \\
\hline CNFC 15010 & 166 & 153 & 119 & 169 & 157 & 84 & 62 \\
\hline CNFP 15208 & 181 & 170 & 168 & 168 & 173 & 46 & 46 \\
\hline IPR Uirapuru & 224 & 172 & 159 & 175 & 179 & 125 & 68 \\
\hline CNFP 15198 & 155 & 159 & 163 & 141 & 170 & 76 & 67 \\
\hline CNFP 15178 & 178 & 181 & 159 & 180 & 170 & 62 & 57 \\
\hline CNFP 15177 & 253 & 182 & 160 & 141 & 156 & 130 & 77 \\
\hline Pérola & 146 & 155 & 177 & 120 & 125 & 74 & 85 \\
\hline BRS CNFC 10425 & 172 & 153 & 164 & 150 & 132 & 116 & 172 \\
\hline BRS CNFC 10762 & 220 & 170 & 173 & 185 & 168 & 103 & 60 \\
\hline CNFC 15025 & 220 & 150 & 142 & 139 & 138 & 93 & 87 \\
\hline BRS Estilo & 320 & 169 & 173 & 161 & 162 & 161 & 92 \\
\hline BRS CNFC 10429 & 349 & 159 & 174 & 151 & 150 & 145 & 126 \\
\hline BRS Campeiro & 404 & 150 & 144 & 165 & 162 & 117 & 87 \\
\hline Total $(\mathrm{sec})$ & 3,625 & 3,268 & 3,244 & 3,191 & 3,215 & 1,789 & 1,342 \\
\hline Total (min) & 60.4 & 54.5 & 54.1 & 53.2 & 53.6 & 29.8 & 22.4 \\
\hline $\mathrm{T}(\%)^{8}$ & - & 90 & 90 & 88 & 89 & 49 & 37 \\
\hline
\end{tabular}

Using a different approach, but also based on digital images, Gray et al. (2013) and Rowland et al. (2015) used the minirhizotron technique to assess nodulation in legumes. Gray et al. (2013) demonstrated the potential of this technique, already used in the observation of root growth, to understand the dynamic of nodule production and distribution in soil profile within the growing season. The estimates generated from minirhizotron for nodule number, density and size were consistent with previously published data for fieldgrown soybean. The use of minirhizotron technique also allowed to study for the first time the nodule formation through the soil profile and across the time in peanut (Rowland et al., 2015).

Cardoso et al. (2009) found out that most of the nodulation resulting from inoculation occurs at the primary crown root of peanut, soybean and common bean. In their study, both in greenhouse and field trials, the crown nodulation (represented by nodule number and dry weight) was generally positively and highly correlated with nodulation of the whole root system for the three legume species. For common bean, the Spearman correlations achieved 0.83 or higher in both environmental conditions.

As observed, most of the studies that tried to reduce the labour involved in nodulation evaluation used devices to obtain digital images (Vikman \& Vessey, 1993; Lira Junior \& Smith, 2000, Lira Junior et al., 2003, 2005; Barbedo, 2012, Gray et al. 2013, Rowland et al. 2015). However, their methods require sophisticated devices and are potentially affected by the uniformity of illumination, the contrast between the nodules and background, etc. Cardoso et al. (2009) also tried to estimate the nodule number, but it is still necessary to use manual counting to obtain the nodule number in the crown root (that corresponds to the region between the cotyledonal node to $7 \mathrm{~cm}$ below). The Sanick, seedburo and, GPR2 methods have high accuracy. Also, GPR2 use low-cost materials and it is not necessary any manual counting. Besides that, there is a great reduction of the time spent for the evaluation.

Although other works inferred that the limitation of time inherent to the conventional determination of the nodulation might have been reduced (Lira Junior \& Smith, 2000; Cardoso et al., 2009), this is the first study that the time was really considered and assessed. Our results showed that all the six alternative methods are efficient in reducing time when compared to manual counting. Even for the worst methods concerning time (PDr1 and PDr2) there was a significant reduction of $10 \%$ when compared to manual counting. This relative short reduction in time is important since in breeding programs there are usually a great number of samples being evaluated.

Concerning time spent, for the genotype BRS Campeiro showing the largest nodule number (Table 1), an evaluator would consume 404 seconds, about 6.7 minutes (Table 3) to count it by MC method. Using the seed counter Sanick, this time would be shortened to 87 seconds or 1.45 minute (Table 3 ), representing a time-saving of about $80 \%$. Time-saving is really important since literature reports studies in which were observed high nodule number, around 271 nodules per plant (Valadão et al., 2009) or large sample number, like in Fonseca et al. (2013) where the sampling consisted of 10 plants per plot. With regard to the time spent on counting, seed counters Sanick and Seedburo stood out, saving 37 and $49 \%$, respectively, of the time compared to MC method. Estimating methods can be faster than the MC method in about $89 \%$.

\section{Materials and Methods}

Plant material, soil type and, site description

Field trials were conducted, with different common bean lines. Treatments consisted of nine "carioca" common bean genotypes (BRS CNFC 10425, BRS CNFC 10429, BRS 
CNFC 10762, BRS Estilo, CNFC 15010, CNFC 15025, CNFC 15082, GEN P5-4-3-1 and Pérola) and 11 "black" common bean genotypes (BRS Campeiro, CNFP 15175, CNFP 15177, CNFP 15178, CNFP 15188, CNFP 15193, CNFP 15194, CNFP 15198, CNFP 15207, CNFP 15208 and IPR Uirapuru). The experiments were carried out under field conditions during the winter season at the experimental area of EMBRAPA Rice and Beans, located in the municipality of Santo Antônio de Goiás, Goiás, Brazil. The chemical soil analysis before sowing showed the following values: $\mathrm{pH}$ $\left(\mathrm{H}_{2} 0\right)$ 6.0, $0 \mathrm{mmol}_{\mathrm{c}} \mathrm{Al} \mathrm{dm}{ }^{-3}, 28 \mathrm{mmol}_{\mathrm{c}} \mathrm{H}+\mathrm{Al} \mathrm{dm}{ }^{-3}, 19$ $\mathrm{mmol}_{\mathrm{c}} \mathrm{Ca} \mathrm{dm}^{-3}, 10 \mathrm{mmol}_{\mathrm{c}} \mathrm{Mg} \mathrm{dm}^{-3}, 9.1 \mathrm{mg} \mathrm{P} \mathrm{dm}^{-3}$ and 87 $\mathrm{mg} \mathrm{P} \mathrm{dm}{ }^{-3}$. The geographical coordinates of the experimental area were $16^{\circ} 30^{\prime} 25^{\prime \prime} \mathrm{S}, 49^{\circ} 16^{\prime} 38^{\prime \prime} \mathrm{W}$ and $799 \mathrm{~m}$ altitude. According to the Köppen classification system, the climate is Aw, tropical savanna, mega thermic. The rainfall regime is well defined, with a rainy season from October to April and a dry season from May to September with an average annual rainfall of $1460 \mathrm{~mm}$ (Silva et al. 2010). The soil in the experimental areas is classified as a clay loam Dystrophic Red Latosol, with clay $=600 \mathrm{~g} \mathrm{~kg}^{-1}$; sand $=130 \mathrm{~g} \mathrm{~kg}^{-1}$ and silt $=270 \mathrm{~g} \mathrm{~kg}^{-1}$.

\section{Field experiment assembling}

The layout of the experiment was a complete randomized block design with four replications. The plot size was $20 \mathrm{~m}^{2}$ $(4 \times 5 \mathrm{~m})$. The trials were fertilized with the recommended $\mathrm{P}_{2} \mathrm{O}_{5}$ and $\mathrm{K}_{2} \mathrm{O}$ fertilization for the crop, with no nitrogen fertilization and the common bean genotypes, were inoculated with Rhizobium strains. For the inoculation, common bean seeds were covered with peat inoculant in the proportion of $500 \mathrm{~g}$ of inoculum per $50 \mathrm{~kg}$ of seeds. The inoculant contained a mixture of Rhizobium tropici (SEMIA 4077 and 4088) and $R$. freirei (SEMIA 4080) strains, in a 1:1:1 proportion of each strain. The final concentration of viable cells in the inoculant was $10^{9}$ cells $\mathrm{g}^{-1}$.

\section{Harvest and manual counting of the nodule number}

For the determination of the nodule number, the harvest of the plants was done only in the inoculated treatments. At the R6 phenological stage, which corresponds to the anthesis (Fernández et al., 1986) three plants with roots were collected from each plot with a straight shovel. The roots were washed in running water and dried under shade during 24 hours. The nodules were carefully detached from the roots and taken to a forced air circulation oven for 36 hours at $65^{\circ} \mathrm{C}$. All nodules of each genotype were manually counted (MC) and weighed to determine the nodule dry weight.

\section{Estimation methods for nodule number}

Four estimation methods were used: graph paper with sampling range from 10 to 20 nodules (GPR1) and graph paper with sampling range from 21 to 40 nodules (GPR2), Petri dish with sampling range from10 to 20 nodules (PDR1), Petri dish with sampling range from 21 to 40 nodules (PDR2). The graph paper method consists of an A4 paper sheet containing squares of $3 \mathrm{~mm}$ x $3 \mathrm{~mm}$ (Figure 1A). Nodules of each genotype were placed on the graph paper, stirred to randomize the size, grouped in the middle in a longitudinal row and, with a ruler, approximately half of nodules was removed from the graph paper (Figure 1B). This procedure was repeated until remain on the graph paper a number of nodules in agreement with the sampling range (between 10 to 20 and, in another moment, from 21 to 40 nodules). These nodules remained on the graph paper were counted and weighed on a precision scale. The dish method consists of a Petri dish of $8 \mathrm{~cm}$ radius $x 1.5 \mathrm{~cm}$ height, which was divided into eight quadrants numbered from 1 to 8 and, each of them, split in half, denominated a and b (Figure 1C). The nodules of each genotype were placed into the Petri dish and stirred to be randomized in all the quadrants (Figure 1D). The nodules were one by one counted and removed from the dish, starting from quadrant 1 section a, following to $1 \mathrm{~b}$, and so on until reaching a number of nodules that were within the sampling ranges (between 10 to 20 and, in another moment, from 21 to 40 nodules). These nodules were weighed on a precision scale. With the nodule number obtained using the dish and the graph paper, its weight and the total weight of the nodules, it was estimated the total nodule number by calculations.

\section{Automated counting methods of nodule number}

The determination of the nodule number by automated counting was performed using two seed counters, Seedburo 801 count-a-pak ${ }^{\circledR}$ and Sanick ESC $2011^{\circledR}$. The nodules were placed in the feeder bowl, which has a spirally inclined track around the inside perimeter. Nodules are moved upward along it by electromagnetic vibration. The reading is done by interruption of the light beam in a chute, located on the top of the track. Seedburo was set to sensitivity 20 and speed 50; and Sanick to speed 60, small seed, target value continuous, mobile knife and stationary delimiter. The time spent for the determination of the nodules of each genotype by each one of the seven evaluation methods (MC, GPR1, GPR2, PDR1, PDR2, Sanick and, Seddburo) was recorded. It was not considered the time spent on sample preparation, nodule detaching and root drying since they were common activities to all counting/estimation methods.

\section{Statistical analyses}

The $X^{2}$ test was performed to nodule number considering the expected frequency as the number of nodules obtained by MC and the observed frequency as the data obtained by the counting/estimation methods. The $X^{2}$ test was also performed to compare the total time spent on estimation or automated counting with the standard method of manual counting for each genotype.

It was calculated the error (\%) obtained in each sample for the counting/estimation methods in relation to the MC:

$$
\mathrm{E}=\left(\frac{|A-M|}{M}\right) * 100 \text {, on what: }
$$

A: nodule number obtained by the counting/estimation methods (estimation or seed counters);

$M$ : nodule number obtained by MC. Then the average was calculated, obtaining, this way, the mean error for each of the six methods. Pearson and Spearman correlations were estimated between the nodule number derived from the MC and from the counting/estimation methods. It was used t test $(\alpha=0.05)$ to test the null hypothesis that there is no correlation between $\mathrm{MC}$ and the other six alternative methods. It was calculated the coincidence between the samples that would be classified as the highest nodule number by the different methods compared with the MC. This test verified if using the alternative methods, and assuming that the samples were different common bean lines being evaluated for BNF performance, they would be selected the same lines as the ones with the highest nodule number. It was considered the intensity selection of $25 \%$, 
which means, the five samples with the highest nodule number.

It was calculated the coincidence index (\%), by Hamblin \& Zimmermann (1986):

$\mathrm{CI}=\left(\frac{A-C}{M-C}\right) * 100$, on what:

$C$ : number of superior samples selected by chance. It is assumed that from the number of superior samples selected, a proportion equal to the intensity selection match by chance, so: if from the 20 samples, it was decided to select $25 \%$ (five samples), $25 \%$ of these (1.25) match by chance;

$A$ : number of superior samples selected in common by MC method and another one;

$M$ : number of superior samples selected (five).

\section{Conclusion}

The results of this work show that alternative methods can be used to assess nodule number in common bean and can be extended to other legumes. The automated counting methods, using the Seedburo and Sanick counters, show high accuracy and reduce at least $50 \%$ of the time spent on counting when compared to manual counting method. Among the methods of estimation, the graph paper with sampling range between 21-40 nodules shows good accuracy with $10 \%$ reduction in time spent when compared to manual counting method, being a good alternative for situations where automated counters are not available. Finally, these results will be helpful for selection programs of plant genotypes and/or rhizobial strains in which the amount of data is extremely large.

\section{Acknowledgements}

To CNPq (Conselho Nacional de Desenvolvimento Científico e Tecnológico) for the fellowship in technology development and innovatory extension to the second, third and fourth authors. To CAPES (Coordenação de Aperfeiçoamento de Pessoal de Nível Superior) for the Ph.D scholarship to the first author.

\section{References}

Albuquerque HC, Pegoraro RF, Vieira NMB, Amorim IJF, Kondo MK (2012) Nodulor capability and agronomic characteristics of common bean plants subjected to fragmented molybdenum and nitrogen fertilization. Rev Cienc Agronom. 43(2):214-221.

Barbedo JGA (2012) Method for automatic counting root nodules using digital images. In: $12^{\text {th }}$ International Conference on Computational Science and Its Applications, 159-161. Murgante B, Gervasi O, Misra S, Nedjah N, Rocha AMAC, Taniar D, Apduhan BO (Eds). Berlin: Springer.

Barros RLN, Oliveira LB, Magalhães WB, Médici LO, Pimentel C (2013) Interaction between rizobial inoculation and sowing nitrogen fertilization on yield of common bean crop at dry and rainy seasons. Semin-Cienc Agrar. 34(4):1443-1450.

Cardoso JD, Gomes DF, Goes KCGP, Fonseca Junior NS, Dorigo OF, Hungria M, Andrade DS (2009) Relationship between total nodulation and nodulation at the root crown of peanut, soybean and common bean plants. Soil Biol Biochem. 41(8):1760-1763.

Costa JVT, Lira Junior MA, Ferreira RLC, Stamford NP, Campanharo M, Sousa CA (2007) Relacionamento entre tamanho do nódulo e medições convencionais da nodulação. Acta Sci-Agron. 29(1):47-54.
Dall'Agnol RF, Ribeiro RA, Ormeño-Orrillo E, Rogel MA, Delamuta JR, Andrade DS, Martínez-Romero E, Hungria M (2013) Rhizobium freirei sp. nov., a symbiont of Phaseolus vulgaris that is very effective at fixing nitrogen. Int J Syst Evol Micr. 63:4167-4173.

Fernández CF, Gepts P, López M. (1986) Etapas de desarrollo de la planta de frijol comun (Phaseolus vulgaris L.). Cali: CIAT. 34 p.

Ferreira NA, Arf O, Carvalho MAC, Araújo RS, Sá ME, Buzetti S (2000) Rhizobium tropici strains for inoculation of the common bean. Sci Agr. 57(3):507-512.

Fonseca GG, Oliveira DP, Soares BL, Ferreira PAA, Teixeira CM, Martins FAD, Moreira FMS, Andrade MJB (2013) Common bean cultivars response to seed inoculation with two rhizobium strains. Biosci J. 29(6):1778-1787.

Gray SB, Strellner RS, Puthuval KK, Ng C, Sullman RE, Siebers MH, Rogers A, Leakey ADB (2013) Minirhizotron imaging reveals that nodulation of field-grown soybean is enhanced by free-air $\mathrm{CO}_{2}$ enrichment only when combined with drought stress. Funct Plant Biol. 40(2):137-147.

Hamblin J, Zimmermann MJO (1986) Breeding common bean for yield in mixtures. In: Plant Breeding Reviews, volume 4 (ed J. Janick), John Wiley \& Sons, Inc., Hoboken, NJ, USA.

Lira Junior MA, Costa C, Smith DL (2003) Effects of addition of flavonoid signals and environmental factors on nodulation and nodule development in the pea (Pisum sativum)-Rhizobium leguminosarum bv. viciae symbiosis. Aust J Soil Res. 41(2):267-276.

Lira Junior MA, Lima AST, Arruda JRF, Smith DL (2005) Effect of root temperature on nodule development of bean, lentil and pea. Soil Biol Biochem. 37(2):235-239.

Lira Junior MA, Smith DL (2000) Use of a standard TWAIN scanner and software for nodule number determination on different legume species. Soil Biol Biochem. 32(10):14631467.

Mhamdi R, Ardley J, Tian R, Seshadri R, Reddy TBK, Pati A, Woyke T, Markowitz V, Ivanova N, Kyrpides N, Reeve W (2015) High-quality permanent draft genome sequence of Ensifer meliloti strain 4H41, an effective salt- and drought-tolerant microsymbiont of Phaseolus vulgaris. Stand Genom Sci. 10(34):1-7.

Novais RF, Alvarez VVH, Barros NF, Fontes RLF, Cantarutti RB, Neves JCL (2007) Fertilidade do solo. 1017 p. Viçosa: Sociedade Brasileira de Ciência do Solo.

Pelegrin R, Mercante FM, Otsubo IMN, Otsubo AA (2009) Response of common bean crop to nitrogen fertilization and rhizobium inoculation. Rev Bras Cienc Solo. 33(1):219-226.

Rowland DL, Smith C, Cook A, Mason A, Schreffler A, Bennett J (2015) Visualization of peanut nodules and seasonal nodulation pattern in different tillage systems using a minithizotron system. Peanut Sci. 42 (1):1-10.

Silva SC, Heinemann AB, Paz RLF, Amorim AO (2010) Informações meteorológicas para pesquisa e planejamento agrícola, referentes ao ano de 2009, do município de Santo Antônio de Goiás, GO. Santo Antônio de Goiás, Brazil: Embrapa Arroz e Feijão.

Valadão FCA, Jakelaitis A, Conus LA, Borchartt L, Oliveira AA, Valadão Junior DD (2009) Seeds inoculation and nitrogen and molybdenum fertilization of common bean in Rolim de Moura, RO. Acta Amaz. 39(4):741-748.

Vikman P, Vessey K (1993) Ontogenetic changes in root nodule subpopulations of common bean (Phaseolus vulgaris L.): III. Nodule formation, growth and degradation. J Exp Bot. 44(260):579-586. 